\title{
Winning strong games through fast strategies for weak games
}

\author{
Asaf Ferber \\ School of Mathematical Sciences \\ Raymond and Beverly Sackler Faculty of Exact Sciences \\ Tel Aviv University, Tel Aviv, 69978, Israel. \\ ferberas@post.tau.ac.il \\ Dan Hefetz \\ School of Mathematical Sciences \\ Queen Mary University of London \\ Mile End Road, London E1 4NS, England. \\ d.hefetz@qmul.ac.uk \\ Submitted: Mar 8, 2011; Accepted: Jun 27, 2011; Published: Jul 15, 2011 \\ Mathematics Subject Classification: 05C57, 05C45, 05C70
}

\begin{abstract}
We prove that, for sufficiently large $n$, the first player can win the strong perfect matching and Hamilton cycle games. For both games, explicit winning strategies of the first player are given. In devising these strategies we make use of the fact that explicit fast winning strategies are known for the corresponding weak games.
\end{abstract}

\section{Introduction}

Let $X$ be a finite set and let $\mathcal{F} \subseteq 2^{X}$ be a family of subsets of $X$. In the strong game $(X, \mathcal{F})$, two players, called Red and Blue, take turns in claiming one previously unclaimed element of $X$, with Red going first. The winner of the game is the first player to fully claim some $F \in \mathcal{F}$. If neither player is able to fully claim some $F \in \mathcal{F}$ by the time every element of $X$ has been claimed by either player, the game ends in a draw. The set $X$ will be referred to as the board of the game and the elements of $\mathcal{F}$ will be referred to as the winning sets.

It is well known from classic Game Theory that, for every strong game $(X, \mathcal{F})$, either Red has a winning strategy (that is, he is able to win the game against any strategy of Blue) or Blue has a drawing strategy (that is, he is able to avoid losing the game against any strategy of Red; a strategy stealing argument shows that Blue cannot win the game). 
For certain games, a hypergraph coloring argument can be used to prove that draw is impossible and thus these game are won by Red. Unfortunately, not much more is known about strong games. In particular, an explicit winning (or drawing) strategy is known only in rare cases. We illustrate this with the following example. In the strong game $R G(n, q)$ the board is $E\left(K_{n}\right)$ and the winning sets are all edge sets of copies of $K_{q}$. It is well known that $R(3)=6, R(4)=18$ and $R(5) \leq 49$, where $R(q)$ is the diagonal Ramsey number (see, e.g., [5]). Hence, the games $R G(6,3), R G(18,4)$ and $R G(49,5)$ cannot end in a draw and are thus a first player's win by strategy stealing. An explicit winning strategy of the first player is known for $R G(6,3)$, but not for $R G(18,4)$ or $R G(49,5)$. Finding such a strategy for $R G(18,4)$ or $R G(49,5)$ is an open problem in [1], where the latter is expected there to be "hopeless".

Partly due to the great difficulty of studying strong games, weak games were introduced. In the weak game $(X, \mathcal{F})$ (more commonly known as a Maker-Breaker game) two players, called Maker and Breaker, take turns in claiming one previously unclaimed element of $X$, with Maker going first (sometimes we will assume that Breaker starts the game; whenever we do, it will be stated explicitly). The game ends as soon as every element of $X$ is claimed by either player. Maker wins the game $(X, \mathcal{F})$ if, by the end of the game, he is able to fully claim some $F \in \mathcal{F}$; otherwise Breaker wins this game. Note that draw is impossible by definition. If Maker has a strategy to win this game against any strategy of Breaker, then we say the game is Maker's win; otherwise we say it is Breaker's win. It turns out that weak games are much easier to study than strong games. Indeed, the theory of weak games is highly developed (the reader is referred to [1] for further details).

Nonetheless, this paper deals with strong games. The starting point of our study of strong games is the following simple observation.

Observation 1.1 Let $X$ be a finite set, let $\mathcal{F} \subseteq 2^{X}$ be a family of subsets of $X$ and let $n:=\min \{|F|: F \in \mathcal{F}\}$ denote the minimum cardinality of a winning set of $\mathcal{F}$. If Maker (as either first or second player) has a strategy to win the weak game $(X, \mathcal{F})$ in $n$ moves, then Red has a strategy to win the strong game $(X, \mathcal{F})$ in $n$ moves.

Indeed, since Red is the first player, Blue has no time to fully claim a winning set. Red can focus on building rather than be worried with blocking Blue's building attempts.

For example, Maker can build a connected graph in $n-1$ moves (this is easy to see and also follows from [4]) and thus Red has a winning strategy for the corresponding strong game by Observation 1.1.

Surprisingly, this trivial observation is, in some sense, best possible. Indeed, consider the following game. Let $n \geq 3$ be an integer. Let $A=\left\{a_{1}, \ldots, a_{9}\right\}$ and let $\mathcal{F}_{A}=\left\{\left\{a_{1}, a_{2}, a_{3}\right\},\left\{a_{4}, a_{5}, a_{6}\right\},\left\{a_{7}, a_{8}, a_{9}\right\},\left\{a_{1}, a_{4}, a_{7}\right\},\left\{a_{2}, a_{5}, a_{8}\right\},\left\{a_{3}, a_{6}, a_{9}\right\}\right.$, $\left.\left\{a_{1}, a_{5}, a_{9}\right\},\left\{a_{3}, a_{5}, a_{7}\right\}\right\}$. That is, $\left(A, \mathcal{F}_{A}\right)$ is just the game Tic-Tac-Toe. Let $B=$ $\left\{b_{1}, \ldots, b_{2 n-6}\right\}$ and let $\mathcal{F}_{B}=\{F \subseteq B:|F|=n-3\}$. Finally, let $X=A \cup B$ and let $\mathcal{F}=\left\{F_{a} \cup F_{b}: F_{a} \in \mathcal{F}_{A}, F_{b} \in \mathcal{F}_{B}\right\}$. Note that $\mathcal{F}$ is $n$-uniform. It is well known (see, e.g., [1]) and easy to prove that strong Tic-Tac-Toe is a draw whereas weak Tic-Tac-Toe is won by Maker (as the first player) in 4 moves. It follows that, by playing the games 
$\mathcal{F}_{A}$ and $\mathcal{F}_{B}$ in parallel, using his 4 -move winning strategy in the former and playing arbitrarily in the latter, Maker can win the weak game $(X, \mathcal{F})$ in $n+1$ moves (as the first player) by playing his first move in $\mathcal{F}_{A}$ and then responding in the same game in which Breaker plays. On the other hand, Blue can force a draw in the strong game $(X, \mathcal{F})$ by following Breaker's winning strategy (as the second player) for the game $\left(A, \mathcal{F}_{A}\right)$ (Blue cannot prevent Red from fully claiming a winning set of $\mathcal{F}_{B}$, but a draw in $\left(A, \mathcal{F}_{A}\right)$ entails a draw in $(X, \mathcal{F}))$.

Nonetheless, in this paper we consider two natural games which are known to be won very quickly by Maker (within $t+1$ moves, where $t$ denotes the minimum cardinality of a winning set) and transform Maker's winning strategy for these games to a winning strategy of Red in the corresponding strong games. Let $n$ be a positive integer. The board of both games is $E\left(K_{n}\right)$, the edge set of the complete graph on $n$ vertices. Hence, from now on we identify a game only by its family of winning sets.

In the perfect matching game $\mathcal{M}_{n}$ the winning sets are all sets of $\lfloor n / 2\rfloor$ independent edges of $K_{n}$. Note that if $n$ is odd, then such a matching covers all vertices of $K_{n}$ but one.

The following result was proved in [3]:

Theorem 1.2 (Theorem 1.2 in [3]) For sufficiently large n, Maker has a winning strategy for the weak game $\mathcal{M}_{n}$, even if Breaker is the first player. Moreover, if $n$ is odd, then Maker can win this game within $\lfloor n / 2\rfloor$ moves, and if $n$ is even, then Maker can win within $n / 2+1$ moves.

Using this result we can prove the following:

Theorem 1.3 For sufficiently large n, Red has a winning strategy for the strong game $\mathcal{M}_{n}$. Moreover, he can win this game within $\lfloor n / 2\rfloor$ moves if $n$ is odd and within $n / 2+2$ moves if $n$ is even.

In the Hamilton cycle game $\mathcal{H}_{n}$ the winning sets are all edge sets of Hamilton cycles of $K_{n}$.

It was proved in [2] that Maker can win the weak game $\mathcal{H}_{n}$ within $n+1$ moves. Here however, we will use the following slightly weaker result from [3]:

Theorem 1.4 (Theorem 1.1 in [3]) For sufficiently large n, Maker has a winning strategy for the weak game $\mathcal{H}_{n}$, even if Breaker is the first player. Moreover, Maker can win this game within $n+2$ moves.

Using this result we can prove the following:

Theorem 1.5 For sufficiently large n, Red has a winning strategy for the strong game $\mathcal{H}_{n}$. Moreover, he can win this game within $n+2$ moves. 
Note that in both games $\mathcal{M}_{n}$ and $\mathcal{H}_{n}$ our strategy for Red requires one more move than the fastest strategies for Maker. We discuss this point further in Section 4.

The rest of this paper is organized as follows: in Subsection 1.1 we introduce some notation and terminology that will be used throughout this paper. In Section 2 we prove Theorem 1.3 and in Section 3 we prove Theorem 1.5. Finally, in Section 4 we present some open problems.

\section{$1.1 \quad$ Notation and terminology}

Our graph-theoretic notation is standard and follows that of [5]. In particular, we use the following.

For a graph $G$, let $V(G)$ and $E(G)$ denote its sets of vertices and edges respectively. Let $\Delta(G)$ denote the maximum degree of $G$. For a set $S \subseteq V(G)$, let $G[S]$ denote the subgraph of $G$, induced on the vertices of $S$. For an edge $e \in E(G)$ we denote by $G \backslash e$ the graph with vertex set $V(G)$ and edge set $E(G) \backslash\{e\}$. A graph is called a linear forest if each of its connected components is a path.

Assume that some strong game, played on the edge set of some graph $G$, is in progress. At any given moment during this game, we denote the graph spanned by Red's edges by $R$, and the graph spanned by Blue's edges by $B$. At any point during the game, the edges of $G \backslash(R \cup B)$ are called free. We also denote by $d_{R}(v)$ and $d_{B}(v)$ the degree of a given vertex $v \in V(G)$ in $R$ and in $B$ respectively.

\section{The Perfect Matching Game}

\section{Proof of Theorem 1.3}

Let $n$ be sufficiently large. Assume first that $n$ is odd. Following Maker's strategy whose existence is guaranteed by Theorem 1.2, Red can build an almost perfect matching of $K_{n}$ in $\lfloor n / 2\rfloor$ moves. It follows from Observation 1.1 that Red wins the strong game $\mathcal{M}_{n}$ in $\lfloor n / 2\rfloor$ moves as claimed.

Assume then that $n$ is even. Let $k=n-2\lfloor n / 4\rfloor$ and let $\mathcal{S}_{\mathcal{M}}$ be a winning strategy for Maker in the weak $\mathcal{M}_{k}$ game. Before describing Red's strategy we prove the following simple lemma.

Lemma 2.1 Assume that just before Red's $(n / 2)$ th move in the strong game $\mathcal{M}_{n}$ the following properties hold:

(i) Red's current graph consists of $n / 2-1$ independent edges and two isolated vertices $x$ and $y$.

(ii) There exist two edges uv and wz in Red's graph such that the subgraph of Blue's graph induced on the vertices of $\{u, v, w, z, x, y\}$ consists solely of the edge $x y$.

(iii) There are at least 3 isolated vertices in $B\left[V\left(K_{n}\right) \backslash\{v\}\right]$. 
Then, for sufficiently large $n$, Red wins the strong game $\mathcal{M}_{n}$ within at most 3 additional moves.

Proof In his $(n / 2)$ th move Red claims the edge $x u$. Blue must respond by claiming the edge $y v$, as otherwise Red will claim it in his next move and thus win. Note that, since Blue has previously claimed $x y$, it follows from property (iii) above that, after claiming $y v$, there are still at least 3 isolated vertices in Blue's graph. Hence, Blue cannot win the game in his $(n / 2+1)$ st move. In his $(n / 2+1)$ st move, Red claims the edge $w y$. Since Blue cannot win or claim both $z x$ and $z v$ in his $(n / 2+1)$ st move, Red claims one of them in his $(n / 2+2)$ nd move and thus wins.

In what follows, we present a strategy for Red in the strong game $\mathcal{M}_{n}$ and then prove that, by following it, Red wins the game within $n / 2+2$ moves against any strategy of Blue.

At any point during the game, a vertex $v$ is called distinct if it is isolated in Red's graph but not in Blue's graph. For every $1 \leq i \leq n / 2$, let $D_{i}$ denote the set of all distinct vertices immediately after Red's $i$ th move and let $D_{i}^{\prime}$ denote the set of all distinct vertices immediately after Blue's $i$ th move. Red's strategy consists of several stages.

Stage 1: In his first move, Red claims an arbitrary edge $e_{1}=u v$. Let $f_{1}=u^{\prime} v^{\prime}$ denote the edge claimed by Blue in his first move. In his second move, Red plays as follows. If $e_{1}$ and $f_{1}$ share a vertex, then Red claims an arbitrary free edge $e_{2}$ which is independent of both $e_{1}$ and $f_{1}$; otherwise, he claims a free edge $e_{2}=u^{\prime} w$, for some $w \in V\left(K_{n}\right) \backslash\{u, v\}$. Red then proceeds to Stage 2.

Stage 2: For every $3 \leq i \leq\lfloor n / 4\rfloor$, in his $i$ th move Red claims an edge $e_{i}$ which is independent of his previously claimed edges while making sure that $\left|D_{i}\right|=1$ (we will prove later that this is indeed possible). If $\Delta(B)>1$ holds immediately after Blue's $\lfloor n / 4\rfloor$ th move, then Red skips to Stage M. Otherwise, for every $\lfloor n / 4\rfloor+1 \leq i \leq n / 2-1$, in his $i$ th move Red claims an edge $e_{i}$ which is independent of his previously claimed edges while making sure that $\left|D_{i}\right|=1$. He then proceeds to Stage 3.

Stage 3: Red completes his matching by claiming at most 3 additional edges as outlined in Lemma 2.1 (an explanation of why this can be done will follow shortly).

Stage M: Let $V_{R}$ denote the set of isolated vertices in Red's graph; note that $\left|V_{R}\right|=$ $n-2\lfloor n / 4\rfloor=k$ is even. Playing on $K_{n}\left[V_{R}\right]$, Red follows $\mathcal{S}_{\mathcal{M}}$ and thus builds a perfect matching of $K_{n}\left[V_{R}\right]$ in $k / 2+1$ moves.

It remains to prove that Red can indeed follow all parts of his strategy and that this ensures his win in the strong game $\mathcal{M}_{n}$ within $n / 2+2$ moves. It is obvious that Red can follow Stage 1 of his strategy. The following lemma asserts that he can follow Stage 2 of his strategy (either for $\lfloor n / 4\rfloor-2$ or $n / 2-3$ moves).

Lemma 2.2 For every $2 \leq i \leq n / 2-1$, Red can ensure that, immediately after his ith move (assuming it is played during Stage 2), his graph is a matching consisting of $i$ edges and $\left|D_{i}\right|=1$.

Proof We prove the lemma by induction on $i$. Red's strategy for Stage 1 yields $\left|D_{2}\right|=1$; this settles the case $i=2$. Assume that $D_{i}=\{z\}$ holds for some $2 \leq i \leq n / 2-2$. We 
prove that, in his $(i+1)$ st move, Red can claim an edge which is independent of all of his previously claimed edges while ensuring $\left|D_{i+1}\right|=1$. In his $i$ th move Blue claims some edge $f=x y$; clearly $1 \leq\left|D_{i}^{\prime}\right| \leq 3$ must hold. We distinguish between the three possible cases:

Case 1: $\left|D_{i}^{\prime}\right|=1$. It follows that $d_{R}(x)=1=d_{R}(y)$ and $D_{i}^{\prime}=\{z\}$. Red claims any free edge $u v$ which is independent of all of his previously claimed edges and such that $z \notin\{u, v\}$.

Case 2: $\left|D_{i}^{\prime}\right|=2$. It follows that $D_{i}^{\prime}=\{x, z\}$ (the case $D_{i}^{\prime}=\{y, z\}$ can be handled similarly). Red claims a free edge $x w$, for some $w \in V\left(K_{n}\right) \backslash\{z\}$, which is independent of all of his previously claimed edges.

Case 3: $\left|D_{i}^{\prime}\right|=3$. It follows that $D_{i}^{\prime}=\{x, y, z\}$. Red claims the edge $x z$.

In either case Red's graph consists of $i+1$ independent edges and $\left|D_{i+1}\right|=1$; hence the assertion of the lemma follows.

Red's last moves are played either in Stage 3 or in Stage M. First assume the latter, that is, assume that $\Delta(B)>1$ holds immediately after Blue's $\lfloor n / 4\rfloor$ th move. It follows that Blue cannot build a perfect matching within $n / 2$ moves. At the moment, Red's graph is a matching $M_{1}$ consisting of $\lfloor n / 4\rfloor$ edges. By Lemma 2.2, just before Blue's $\lfloor n / 4\rfloor$ th move there was exactly one distinct vertex. Hence, immediately after Blue's $\lfloor n / 4\rfloor$ th move, there is at most one edge in $B\left[V_{R}\right]$. It follows that, assuming the role of Maker (as the second player) in the weak perfect matching game on $K_{n}\left[V_{R}\right]$, Red can build a perfect matching $M_{2}$ of $K_{n}\left[V_{R}\right]$ within $k / 2+1$ moves. Note that $M_{1} \cup M_{2}$ is a perfect matching of $K_{n}$. Moreover, Red built this matching within $\lfloor n / 4\rfloor+(k / 2+1)=n / 2+1$ moves. Since, as previously noted, Blue cannot build a perfect matching in $n / 2$ moves, it follows that Red wins the strong game.

Next, assume the former, that is, assume that $\Delta(B)=1$ holds immediately after Blue's $\lfloor n / 4\rfloor$ th move. It follows that the following properties hold immediately after Red's $(n / 2-1)$ th move (that is, at the end of Stage 2$)$ :

(a) Red's graph is a matching consisting of $n / 2-1$ edges.

(b) $\left|D_{n / 2-1}\right|=1$.

(c) $|E(B)|=n / 2-2$.

(d) $\Delta(B) \leq\lceil n / 4\rceil-1$

It follows by property $(a)$ above that there are exactly two isolated vertices in Red's graph, say $x$ and $y$. It follows by property $(b)$ above that exactly one of the vertices of $\{x, y\}$ is distinct. Assume without loss of generality that $D_{n / 2-1}=\{x\}$. In his $(n / 2-1)$ th move, Blue must claim the edge $x y$ as otherwise Red will claim it in his $(n / 2)$ th move and thus win. Immediately after Blue's $(n / 2-1)$ th move there are at least 3 isolated vertices in his graph and, moreover, $d_{B}(y)=1$ and $d_{B}(x) \leq\lceil n / 4\rceil$. It follows that there 
exist edges $u v$ and $w z$ in Red's graph such that $x y$ is the only edge of $B[\{u, v, w, z, x, y\}]$. Moreover, if there are exactly 3 isolated vertices in Blue's graph, then clearly one can choose $v$ such that $d_{B}(v) \geq 1$. Hence, all the conditions of Lemma 2.1 are satisfied and therefore Red wins the game within at most 3 additional moves.

This concludes the proof of Theorem 1.3.

\section{The Hamilton Cycle Game}

In our proof of Theorem 1.5 we will make use of Theorem 1.4 and of the specific strategy $\mathcal{S}_{\mathcal{H}}$ that was used in its proof in [3]. Therefore, we begin by providing a rough outline of this strategy (for simplicity of presentation we only consider the case where $n$ is even, the complementary case is similar).

Maker's strategy $\mathcal{S}_{\mathcal{H}}$ consists of the following three stages:

Stage 1: Maker builds a perfect matching with one additional edge, that is, he builds a linear forest which consists of one path of length 3 and $n / 2-2$ paths of length 1 each. This stage lasts exactly $n / 2+1$ moves.

Stage 2: For every $0 \leq i \leq n / 2-3$, let $B_{i}^{\prime}$ be the subgraph of Breaker's graph induced on the endpoints of Maker's paths immediately after Breaker's $i$ th move in Stage 2. Let $B_{i}$ be the graph obtained from $B_{i}^{\prime}$ by removing all edges $x y$ such that $x$ and $y$ are endpoints of the same path in Maker's graph. The free edges $x y \in\left(\begin{array}{c}V\left(B_{i}\right) \\ 2\end{array}\right)$, for which $x$ and $y$ are endpoints of different paths of Maker are called available. For every $1 \leq j \leq n / 2-3$, in his $j$ th move in Stage 2, Maker claims an available edge while making sure that $\left|E\left(B_{j}\right)\right| \leq\left|V\left(B_{j}\right)\right|-1$ will hold immediately after Breaker's $j$ th move in Stage 2. In his $(n / 2-2)$ th move in Stage 2 , Maker connects his two paths to form a Hamilton path. This stage lasts exactly $n / 2-2$ moves.

Stage 3: Maker closes the Hamilton path he has built by the end of Stage 2 into a Hamilton cycle within at most 3 additional moves. This can be done if the maximum degree in Breaker's graph does not exceed $n / 2$.

Note that, in some of his moves in Stage 2, Maker has certain freedom in choosing an edge to claim. This results in the following useful observation.

Observation 3.1 For every $0 \leq i \leq n / 2-3$, if immediately after Breaker's ith move in Stage 2, Maker's graph is a linear forest consisting of $n / 2-i-1$ non-empty paths and $\left|E\left(B_{i}\right)\right| \leq\left|V\left(B_{i}\right)\right|-1$, then Maker can follow $\mathcal{S}_{\mathcal{H}}$ from this point on and win $\mathcal{H}_{n}$ in $n+2$ moves.

Note that, by following Maker's strategy $\mathcal{S}_{\mathcal{H}}$, Red can build a Hamilton cycle within at most $n+2$ moves. This entails the following simple observation.

Observation 3.2 In the strong game $\mathcal{H}_{n}$, if Red follows $\mathcal{S}_{\mathcal{H}}$ and Blue does not build a Hamilton cycle within $n+1$ moves, then Red wins the game. In particular, if Blue does not build a Hamilton path within $n$ moves, then Red wins the game. 
The following two lemmas show that, in order to stand a chance at winning, Blue must in fact build a Hamilton path within $n-1$ moves.

Lemma 3.3 Assume that immediately before Red's nth move in $\mathcal{H}_{n}$ the following properties hold:

(i) Red's graph is a Hamilton path $x_{1} x_{2} \ldots x_{n}$.

(ii) Blue's graph is not a Hamilton path.

(iii) $\Delta(B) \leq 10$.

(iv) There are at most 10 free edges which, if claimed by Blue in his nth move, would form a Hamilton path in his graph.

Then, for sufficiently large $n$, Red wins the strong game $\mathcal{H}_{n}$ within at most 3 additional moves.

Proof In his $n$th move, Red claims a free edge $x_{1} x_{k}$ such that $k \geq n / 2$, the edge $x_{k-1} x_{n}$ is free and its addition to Blue's graph does not form a Hamilton path in it. Such an edge $x_{1} x_{k}$ exists by properties (iii) and ( $i v$ ) above and since $n$ is assumed to be sufficiently large. It follows by property (ii) that Blue cannot win the game in his $n$th move. Hence, we can assume that Blue claims $x_{k-1} x_{n}$ in his $n$th move as otherwise Red will claim this edge in his $(n+1)$ st move and thus win. Note that, by our choice of $x_{1} x_{k}$, immediately after Blue's $n$th move his graph still does not admit a Hamilton path. It follows that Blue will not win the game in his next move either. In his $(n+1)$ st move, Red claims an edge $x_{t} x_{n}$ such that $t<k-2$ and both edges $x_{1} x_{t+1}$ and $x_{t+1} x_{k+1}$ are free. Again this is possible by property (iii) above and since $n$ is assumed to be sufficiently large. Since, as previously noted, Blue cannot win the game in his $(n+1)$ st move, and since he cannot claim both $x_{1} x_{t+1}$ and $x_{t+1} x_{k+1}$ in this move, it follows that Red wins (by claiming one of these edges $)$ in his $(n+2)$ nd move as claimed.

Lemma 3.4 Assume that immediately before Red's nth move in $\mathcal{H}_{n}$ the following properties hold:

(i) Red's graph is a Hamilton path $x_{1} x_{2} \ldots x_{n}$.

(ii) There exists a vertex $x \in V\left(K_{n}\right)$ such that $d_{B}(x) \geq 3$.

(iii) $\Delta(B) \leq 10$.

Then, for sufficiently large $n$, Red wins the strong game $\mathcal{H}_{n}$ within at most 3 additional moves. 
Proof If there are strictly more than two connected components in Blue's graph, then, by Observation 3.2, Red wins the game within 3 additional moves. It remains to consider the following two cases.

Case 1: Blue's graph is connected. Since $|E(B)|=n-1$ it must be a tree. Moreover, by property (ii) above, this tree has at least 3 leaves. If it has strictly more than 4 leaves, then Blue cannot build a Hamilton path by his $n$th move. Hence, by Observation 3.2, Red wins the game within at most 3 additional moves as claimed. If this tree has exactly 4 leaves, then if it is possible for Blue to build a Hamilton path in his $n$th move, then it is by claiming a free edge which connects two such leaves. Clearly there are at most $\left(\begin{array}{l}4 \\ 2\end{array}\right)=6$ such edges. Hence, the conditions of Lemma 3.3 are satisfied and thus Red wins within at most 3 additional moves as claimed. Assume then that the tree $B$ has exactly 3 leaves. Let $a, b, c$ denote these leaves and note that $d_{B}(x)=3$ and $d_{B}(u)=2$ for every $u \in V\left(K_{n}\right) \backslash\{x, a, b, c\}$. Let $P_{a}, P_{b}$ and $P_{c}$ denote the unique paths in $B$ between $x$ and $a, b$ and $c$ respectively. For every $i \in\{a, b, c\}$, let $x^{(i)}$ denote the unique neighbor of $x$ in $P_{i}$. Note that if it is possible for Blue to build a Hamilton path in his $n$th move, then it is by claiming one of the following 9 edges: $a b, a c, b c, a x^{(b)}, a x^{(c)}, b x^{(a)}, b x^{(c)}, c x^{(a)}, c x^{(b)}$. Hence, the conditions of Lemma 3.3 are satisfied and thus Red wins within at most 3 additional moves as claimed.

Case 2: Blue's graph admits exactly 2 connected components. Let $C_{1}$ and $C_{2}$ be these two components, where $x \in V\left(C_{1}\right)$. If there exists some $y \in V\left(C_{2}\right)$ such that $d_{B}(y) \geq 3$, then, by Observation 3.2, Red wins the game within 3 additional moves. Hence, we can assume that $d_{B}(u) \leq 2$ for every $u \in V\left(C_{2}\right)$. It follows that $C_{2}$ is either a path or a cycle. If $C_{2}$ is a cycle, then Blue has "wasted" at least two edges in his previous moves (one for closing this cycle and the other since $d_{B}(x) \geq 3$ ). Hence, by Observation 3.2, Red wins the game within at most 3 additional moves as claimed. Assume then that $C_{2}$ is a path; it follows that $C_{1}$ is a tree with one additional edge. Note that $C_{1}$ is not a cycle as it admits a vertex of degree at least 3 . By Observation 3.2 we can assume that $d_{B}(x)=3$ and that there exists a vertex $z \in C_{1}$ such that $C_{1} \backslash x z$ is a tree with maximum degree 2 , that is, a path. It follows that there are at most 6 free edges whose addition to Blue's graph creates a Hamilton path in it. Hence, the conditions of Lemma 3.3 are satisfied and thus Red wins the game within at most 3 additional moves as claimed.

Proof of Theorem 1.5: Assume first that $n$ is even. We start by describing Red's strategy; it consists of several stages.

Stage 1: In his first $n / 2+1$ moves, Red follows $\mathcal{S}_{\mathcal{H}}$.

Stage 2: For every $0 \leq i \leq n / 2-3$, let $B_{i}^{\prime}$ be the subgraph of Blue's graph induced on the endpoints of Red's paths immediately after Blue's $i$ th move in Stage 2 . Let $B_{i}$ be the graph obtained from $B_{i}^{\prime}$ by removing all edges $x y$ such that $x$ and $y$ are endpoints of the same path in Red's graph. The free edges $x y \in\left(\begin{array}{c}V\left(B_{i}\right) \\ 2\end{array}\right)$, for which $x$ and $y$ are endpoints of different paths of Red are called available. For every $1 \leq i \leq n / 2-2$, in his $i$ th move of Stage 2, Red claims some available edge. Before each of his moves in this stage, Red checks if the following conditions hold:

(i) $\Delta(B) \geq 3$. 
(ii) There are at least two cycles in Blue's graph.

If at least one of these two conditions is satisfied, then Red proceeds to Stage M, otherwise he plays another move in Stage 2.

Stage 2 is divided into the following three sub-stages:

(2.1) In at most 3 moves, Red makes sure that there exists a vertex $x \in V\left(K_{n}\right)$ with the following properties:

(a1) $d_{R}(x)=1$.

(a2) $d_{B}(x)=2$.

(a3) Both neighbors of $x$ in Blue's graph have degree 2 in Red's graph.

(2.2) Let $P_{x}$ denote Red's path with $x$ as an endpoint. Let $y$ denote the other endpoint of $P_{x}$. For as long as Red's graph consists of at least 3 connected components, he plays as follows. In his $i$ th move of Stage 2, Red claims an available edge $u v$ while making sure that the following properties hold immediately after Blue's $i$ th move:

(b1) $\left|E\left(B_{i}\right)\right| \leq\left|V\left(B_{i}\right)\right|-1$.

(b2) $\{x, y\} \cap\{u, v\}=\emptyset$.

(b3) If in his $(i-1)$ th move of Stage 2, Blue claims an available edge $y w$ for some $w \in V\left(B_{i-1}\right)$, then Red claims an available edge $u w$ while maintaining properties (b1) and (b2) above.

(2.3) Red connects his two paths to form a Hamilton path, while making sure that the conditions of Lemma 3.4 are satisfied.

Stage 3: Red closes the Hamilton path he has built by the end of Stage 2 into a Hamilton cycle within at most 3 additional moves.

Stage M: From this point on, Red follows $\mathcal{S}_{\mathcal{H}}$ until his graph is a Hamilton path. Then, if $\Delta(B)>3$, Red continues playing according to $\mathcal{S}_{\mathcal{H}}$, otherwise he plays as outlined in Lemma 3.4.

It remains to prove that Red can indeed follow all parts of his strategy and that this ensures his win in the strong game $\mathcal{H}_{n}$ within $n+2$ moves.

Stage 1: This follows immediately from the proof of Theorem 1.4 in [3]. It follows that, by the end of this stage, Red's graph is a linear forest which consists of $n / 2-1$ vertex disjoint paths, where one path is of length 3 and the rest are of length 1 each.

Stage 2: We consider each sub-case separately.

(2.1) We know that $\Delta(B) \leq 2$ holds throughout this sub-stage, since we are diverted to Stage $\mathrm{M}$ as soon as $\Delta(B) \geq 3$. Assume first that there exists a vertex $x \in V\left(B_{0}\right)$ such that $d_{B}(x)=2$. Let $u$ and $v$ be the two neighbors of $x$ in Blue's graph. For every $w \in\{u, v\}$, if $d_{R}(w)=1$, then Red claims some available edge which is incident with $w$. This takes at most 2 moves. Next, assume that no such vertex $x$ exists in $V\left(B_{0}\right)$. Let $z_{1}$ and $z_{2}$ denote the only two vertices of degree 2 in Red's graph. Since Blue has also 
claimed $n / 2+1$ edges, his graph must admit at least two vertices of degree 2 as well. We can assume that there are exactly two such vertices and that they are in fact $z_{1}$ and $z_{2}$, as otherwise we are in the previous case. It follows that Blue's graph is a linear forest consisting of two paths of length 2 each and $n / 2-3$ paths of length 1 each. It follows that there are no isolated vertices in Blue's graph. In his first move in Stage 2, Red claims an arbitrary available edge $w_{1} w_{2}$. Note that $d_{R}\left(w_{1}\right)=d_{R}\left(w_{2}\right)=2$ holds after this move. In his first move in Stage 2, Blue clearly cannot claim an edge which is incident with both $w_{1}$ and $w_{2}$. Hence, after this move, there must exist a vertex $x \in V\left(B_{1}\right)$ such that $d_{B}(x)=2$. Red now "takes care" of the neighbors of $x$ in Blue's graph, as in the previous case. This takes Red at most 3 moves.

(2.2) Assume that we are just before Red's $i$ th move in Stage 2 and that so far Red was able to maintain Properties (b1), (b2) and (b3); note that, assuming $n$ is sufficiently large, these properties hold at the beginning of this stage. Since we are not in Stage M we know that $\Delta(B) \leq 2$. In his $i$ th move, Red claims some available edge $w z$ while ensuring that Properties (b2) and (b3) are maintained; this is clearly possible. In his $i$ th move, Blue claims an arbitrary edge $e$; again we know that $\Delta(B) \leq 2$ still holds after this move as otherwise we are diverted to Stage M. Note that, regardless of Blue's $i$ th move, $V\left(B_{i}\right)=V\left(B_{i-1}\right) \backslash\{w, z\}$. Since Red maintained Property (b2), it follows that $\{x, y\} \subseteq V\left(B_{i}\right)$. Since Red maintained Property (b3), it follows that, immediately before Blue's $i$ th move, both $x$ and $y$ are isolated in $B_{i} \backslash e$. Since $\Delta(B) \leq 2$, it follows that $\left|E\left(B_{i} \backslash e\right)\right| \leq\left|V\left(B_{i}\right)\right|-2$. Hence, immediately after Blue's $i$ th move, Property (b1) will be satisfied as well.

(2.3) Let $u$ and $v$ denote the endpoints of Red's only path other than $P_{x}$. If, in his $(n-2)$ th move, Blue claims an edge $x w$ for some $w \in V\left(K_{n}\right)$, then $d_{B}(x) \geq 3$ and thus Red proceeds to Stage M. Hence, assume that Blue does not claim such an edge in his $(n-2)$ th move. Note that, by Red's strategy, just before his $(n-1)$ th move both edges $x u$ and $x v$ are free. Moreover, at least one of the edges $y u$ and $y v$ is free. Assume without loss of generality that $y u$ is free. In his $(n-1)$ th move, Red claims yu thus completing a Hamilton path. In his $(n-1)$ th move, Blue must claim $x v$ as otherwise Red will claim it in his $n$th move and thus win. It follows that just before Red's $n$th move, the conditions of Lemma 3.4 are satisfied.

Stage 3: Red plays as outlined in Lemma 3.4 and thus wins within at most 3 additional moves.

Stage M: If Red skipped to this stage before making any move in Stage 2, then it is clear he can continue playing according to $\mathcal{S}_{\mathcal{H}}$. Assume then that Red skipped to Stage M immediately after Blue's $i$ th move in Stage 2 , for some $i \geq 1$. It follows by Stage (2.2) of Red's strategy that $\left|E\left(B_{i}\right)\right| \leq\left|V\left(B_{i}\right)\right|-1$ was true immediately after Blue's $i$ th move in Stage 2. Hence, by Observation 3.1, Red can continue playing according to $\mathcal{S}_{\mathcal{H}}$ until his graph is a Hamilton path. Since $\Delta(B) \geq 3$, he can then win by either Lemma 3.4 or Observation 3.2 in at most 3 additional moves.

If $n$ is odd, then the proof is essentially the same. We list below the main differences from the case of even $n$.

(1) As in [3], during Stage 1 Red builds a linear forest which consists of one path of length 
2 and $\lfloor n / 2\rfloor-1$ paths of length 1 each. This stage lasts exactly $\lfloor n / 2\rfloor+1$ moves.

(2) At the beginning of Stage (2.1), there is exactly one vertex of degree 2 in Red's graph (the rest are of degree exactly 1) and at least one vertex of degree 2 in Blue's graph. Hence, as in the case of even $n$, either there exists a vertex $x \in V\left(B_{0}\right)$ such that $d_{B}(x)=2$, or Red makes sure there will be such a vertex in $V\left(B_{1}\right)$.

This concludes the proof of Theorem 1.5.

\section{Concluding remarks and open problems}

Strong wins via fast weak wins for more games. We have seen how known fast winning strategies for Maker in the perfect matching game $\mathcal{M}_{n}$ and the Hamilton cycle game $\mathcal{H}_{n}$ can be used to devise winning strategies for Red in the corresponding strong games. It seems plausible that the same idea could be used for other games as well. A natural candidate is the $k$-vertex-connectivity game, played on $E\left(K_{n}\right)$. Indeed, as noted in the introduction, Maker can build a connected graph in $n-1$ moves and thus Red has a winning strategy for the corresponding strong game by Observation 1.1. Moreover, it is not hard to see that, by following his strategy for the Hamilton cycle game (given in the proof of Theorem 1.5), Red builds a 2-connected graph within $n+1$ moves. Since a cycle on $n$ vertices is the only 2-connected subgraph of $K_{n}$ with $n$ edges, and since Blue cannot build one in just $n$ moves by Theorem 1.5, it follows that Red wins this game as well. For $k \geq 3$ it was proved in [3] that Maker can build a $k$-vertex-connected graph within $k n / 2+(k+4)\left(\sqrt{n}+2 n^{2 / 3} \ln n\right)$ moves. It was also asked there whether there exists a function $f$ such that Maker can in fact win this game within $k n / 2+f(k)$ moves. Answering the latter question in the affirmative might help in devising a winning strategy for Red in the strong $k$-vertex-connectivity game.

Quickness vs. Initiative. As noted in the introduction, while the known strategies for Maker allow him to win $\mathcal{M}_{n}$ within $\lfloor n / 2\rfloor$ moves if $n$ is odd and within $n / 2+1$ moves if $n$ is even, and to win $\mathcal{H}_{n}$ within $n+1$ moves (all of these results are best possible), our strategies for Red require $n / 2+2$ moves to win $\mathcal{M}_{n}$ if $n$ is even and $n+2$ moves to win $\mathcal{H}_{n}$ (we do not know if these bounds are best possible or not). One reason for this discrepancy is that the fastest strategies for Maker force him to "waste" a move early on (in the strategy for $\mathcal{M}_{n}$ given in [3], either the first or second edge Maker claims will not be part of his matching; in the strategy for $\mathcal{H}_{n}$ given in [2], it is not so clear when Maker will claim an edge which will not be part of his Hamilton cycle, but this could happen very early in the game). While this proves useful for Maker, it might be dangerous for Red as it makes him lose the initiative and allows Blue to create threats. Hence, while we are using in this paper the fact that Maker wins quickly, the crux of the matter is that our strategies for Red preserve his initiative throughout the game. It might be possible (though seems hard) to find such strategies even for games Red cannot win very quickly. 


\section{References}

[1] J. Beck, Combinatorial Games: Tic-Tac-Toe Theory, Cambridge University Press, 2008.

[2] D. Hefetz and S. Stich, On two problems regarding the Hamilton cycle game, The Electronic Journal of Combinatorics 16(1) (2009), R28.

[3] D. Hefetz, M. Krivelevich, M. Stojaković and T. Szabó, Fast winning strategies in Maker-Breaker games, J. of Combinatorial Theory, Ser. B. 99 (2009), 39-47.

[4] A. Lehman, A solution of the Shannon switching game, J. Soc. Indust. Appl. Math. 12 (1964), 687-725.

[5] D. B. West, Introduction to Graph Theory, Prentice Hall, 2001. 